\title{
TGFBR3 variation is not a common cause of Marfan-like syndrome and Loeys-Dietz-like syndrome
}

\author{
Krishna K Singh ${ }^{1,2}$, Joerg Schmidtke ${ }^{1}$, Britta Keyser ${ }^{1}$ and Mine Arslan-Kirchner ${ }^{1 *}$
}

\begin{abstract}
Marfan syndrome (MFS) is caused by mutations in the fibrillin-1 (FBN1) gene, and mutations in FBN1 are known to be responsible for over $90 \%$ of all MFS cases. Locus heterogeneity has also been reported and confirmed, with mutations in the receptor genes TGFBR1 and TGFBR2 identified in association with MFS-related phenotypes. It is now known that dysregulation of TGF- $\beta$ signaling is involved in MFS pathogenesis. To test the hypothesis that dysregulation of TGFBR3-associated TGF-ß signaling is implicated in MFS or related phenotype pathogenesis, we selected a cohort of 49 patients, fulfilling or nearly fulfilling the diagnostic criteria for MFS. The patients were known not to carry a mutation in the FBN1 gene (including three $5^{\prime}$ upstream alternatively spliced exons), the TGFBR1 and TGFBR2 genes. Mutation screening for the TGFBR3 gene in these patients and in controls led to the identification of a total of ten exonic (one novel), four intronic (one novel) and one $3^{\prime} U T R$ variant in the TGFBR3 gene. Our data suggest that variations in TGFBR3 gene appear not to be associated with MFS or related phenotype.
\end{abstract}

Keywords: MFS, LDS, TGFBR3, variants

\section{Background}

Marfan syndrome (MFS; MIM\# 154700) is an autosomal-dominant disorder of connective tissue with major manifestations in the skeletal, cardiovascular and ocular systems. MFS is caused by mutations in the fibrillin-1 gene (FBN1), and mutations in $F B N 1$ are known to be responsible for over $90 \%$ of all MFS cases. However, locus heterogeneity was reported in the early 1990's, when a second locus 3p24.2-p25 was suggested to cause MFS [1]. This association was further confirmed when mutations were identified in the transforming growth factor $ß$ receptor type II gene (TGFBR2), which maps to the corresponding chromosomal region, in patients with overlapping phenotypes of MFS and Loeys-Dietz syndrome (LDS1B; MIM\#610168) [2-4]. Later, using a functional approach, mutations were identified in another receptor of TGF- $B$ receptor family, transforming growth factor $\beta$ receptor type I (TGFBR1) in association with MFS or related phenotypes LDS (LDS1A; MIM\#609192)

\footnotetext{
* Correspondence: arslan.mine@mh-hannover.de

${ }^{1}$ Institute of Human Genetics, Hannover Medical School, Hannover, Germany Full list of author information is available at the end of the article
}

[3-5]. These and other findings, strongly suggested an important role played by TGF- $\beta$ receptors and TGF- $\beta$ signaling dysregulation in the pathogenesis of MFS and related phenotypes $[6,7]$. The TGF- $\beta$ signaling pathway regulates extracellular matrix formation through members of the TGF- $ß$ superfamily and their receptors [8]. TGF- $ß$ mainly functions by binding to three cell surface receptors, namely TGFBR1 (55 kD), TGFBR2 (80 kD) and transforming growth factor receptor type III (TGFBR3, $280 \mathrm{kD}$ ) [9]. TGFBR3 is the most abundantly expressed subtype, has high affinity for all three TGF- $\beta$ isoforms, and acts as an enhancer of the TGF- $\beta$ access to the other signaling receptors [10]. So far, no systematic search for TGFBR3 genetic variation associated with MFS and related phenotypes has been reported in the literature. To test the hypothesis that dysregulation of TGFBR3-associated TGF- $B$ signaling is implicated in MFS or related phenotype pathogenesis, we selected a cohort of 49 patients, fulfilling or nearly fulfilling the diagnostic criteria for MFS. The patients were known not to carry a mutation in the FBN1 gene (including three 5' upstream alternatively spliced exons), the

C Biomed Central

(c) 2012 Singh et al; licensee BioMed Central Ltd. This is an Open Access article distributed under the terms of the Creative Commons Attribution License (http://creativecommons.org/licenses/by/2.0), which permits unrestricted use, distribution, and reproduction in any medium, provided the original work is properly cited. 
TGFBR1 and TGFBR2 genes. Mutation screening for the TGFBR3 gene in these patients and in controls led to the identification of a total of ten exonic (one novel), four intronic (one novel) and a 3'UTR variant in the TGFBR3 gene. Our data suggest that variations in TGFBR3 gene appear not to be associated with MFS or related phenotype.

\section{Results and Discussion}

In a cohort of 49 unrelated probands with the tentative diagnosis of Marfan syndrome or fulfilling criteria of the "revised Ghent nosology" of 1996 [11] without identified mutation in the FBN1, TGFBR2, and TGFBR1 coding regions, a systematic mutation screen was performed by sequencing all 17 exons of TGFBR3 gene. A total of ten exonic (one novel), four intronic (one novel) and a 3'UTR sequence alterations were detected. Molecular findings of all index patients and relatives carrying variants in TGFBR3 gene are summarized in table 1.

Among the exonic variants identified; c.44C $>\mathrm{T}$ (p. S15F; exon 2), c.216G > A (p. A72A; exon3), c.1128 (p. I376I; exon 9), c.1206G > A (p.P402P; exon 9), c.1341C $>\mathrm{T}$ (p.S447S; exon 9), c.2028C > T (p.F676F; exon 13) and c.2247C > T (p.T749T; exon 14) were detected in index patients in the same allele frequency as controls. Bioinformatic analyses using the online-software Mutation Taster, PMut and PolyPhen 2 did not assign any disease-causing effect to these variants. Two already known exonic variants c.2293G > C (p.G765R; exon 15) and c.2329C > T (p.P777S; exon 15) were only detected in two and one index cases respectively, but not in controls. The first index case with the c.2293G > C variant was a 17-year-old male, who fulfilled the Ghent major criterion in the skeletal system, showed the involvement of the cardiovascular system and had a negative family history. The second index case with the c.2293G $>\mathrm{C}$ variant was a male sporadic case with suspected MFS and he was 26 years of age at the time of examination. The skeletal system was involved (body proportions, positive thumb and wrist signs, scoliosis, highly arched palate, typical facial features) and a major criterion would have been fulfilled, if he had been tested positive for the presence of protusio acetabuli. He had mitral valve prolapse. The variant c.2293G > C was present in his mother, who had no signs of MFS, and was absent in the healthy father.

The variant c. $2329 \mathrm{C}>\mathrm{T}$ was identified in a 14-yearold boy with involvements of the skeletal system and the skin. He had normal height at the age of 12-years, a slight funnel chest, flat feet, positive thumb and wrist signs, highly arched palate and joint hypermobility with recurrent herniae. Further anomalies were hypodontia (aplasia of 9 teeth), dysmorphic ears and stenosis of the external auditory meatus. At the age of 13-years celiac disease was diagnosed. His parents did not have signs of MFS, his mother was hypodontic but we were unable to screen the mother for the presence of this variant. This variant was identified in the healthy father.

The online-program PMut predicted both variants, c.2293G > C and c.2329C > T to be possibly pathogenic. On the contrary, the online-software Mutation Taster and PolyPhen 2 did not assign any disease-causing effect

Table 1 Variants identified and their respective allele frequencies in the TGFBR3 gene

\begin{tabular}{|c|c|c|c|c|c|c|}
\hline Variants & Location & Amino Acid & Allele freq. Patient $(n=49)$ & Allele freq. Controls & Ref. Acc. Nr. & Allele freq. \\
\hline C. $44 C>T$ & Exon 2 & S15F & 0.11 & $0.12(n=54)$ & rs1805110 & 0.325 \\
\hline C. $55 \mathrm{~A}>\mathrm{G}$ & Exon 2 & T19A & 0.02 & $0.00(n=54)$ & Novel & \\
\hline c.62-51 C > T & Intron 2 & & 0.01 & $0.03(n=52)$ & rs17881268 & 0.03 \\
\hline c. $216 G>A$ & Exon 3 & A72A & 0.32 & $0.35(n=52)$ & rs2810904 & 0.407 \\
\hline c.247-40C > T & Intron 3 & & 0.18 & $0.13(n=45)$ & rs11165441 & 0.13 \\
\hline c.886-1 OA > G & Intron 7 & & 0.01 & $0.00(n=40)$ & Novel & \\
\hline c. $1128 \mathrm{C}>\mathrm{T}$ & Exon 9 & $1376 \mid$ & 0.01 & $0.00(n=55)$ & rs11466595 & 0.015 \\
\hline c.1206G > A & Exon 9 & P402P & 0.41 & $0.41(n=55)$ & rs1805112 & 0.477 \\
\hline c.1341C > T & Exon 9 & S447S & 0.02 & $0.02(n=55)$ & rs2229500 & ND \\
\hline C. $1566+55 C>A$ & Intron 10 & & 0.26 & $0.28(n=58)$ & rs7524066 & 0.19 \\
\hline c.2028C > T & Exon 13 & F676F & 0.51 & $0.41(n=59)$ & rs1805113 & 0.417 \\
\hline c.2247C > T & Exon 14 & T749T & 0.07 & $0.07(n=50)$ & rs284878 & 0.196 \\
\hline c.2293G > C & Exon 15 & G765R & 0.02 & $0.00(n=50)$ & rs17882828 & 0.034 \\
\hline c. $2329 C>T$ & Exon 15 & P777S & 0.01 & $0.00(n=50)$ & rs35352606 & 0.01 \\
\hline$\overline{c .}{ }^{*} 19 \mathrm{G}>\mathrm{A}$ & 3'UTR & & 0.13 & $0.25(n=52)$ & rs1131243 & 0.10 \\
\hline
\end{tabular}

The numbering is based on the mRNA sequence (TGFBR3; accession number NM_003243.4), where 1 corresponds to the nucleotide A of ATG, the translation initiation codon.

ND; not yet determined. 


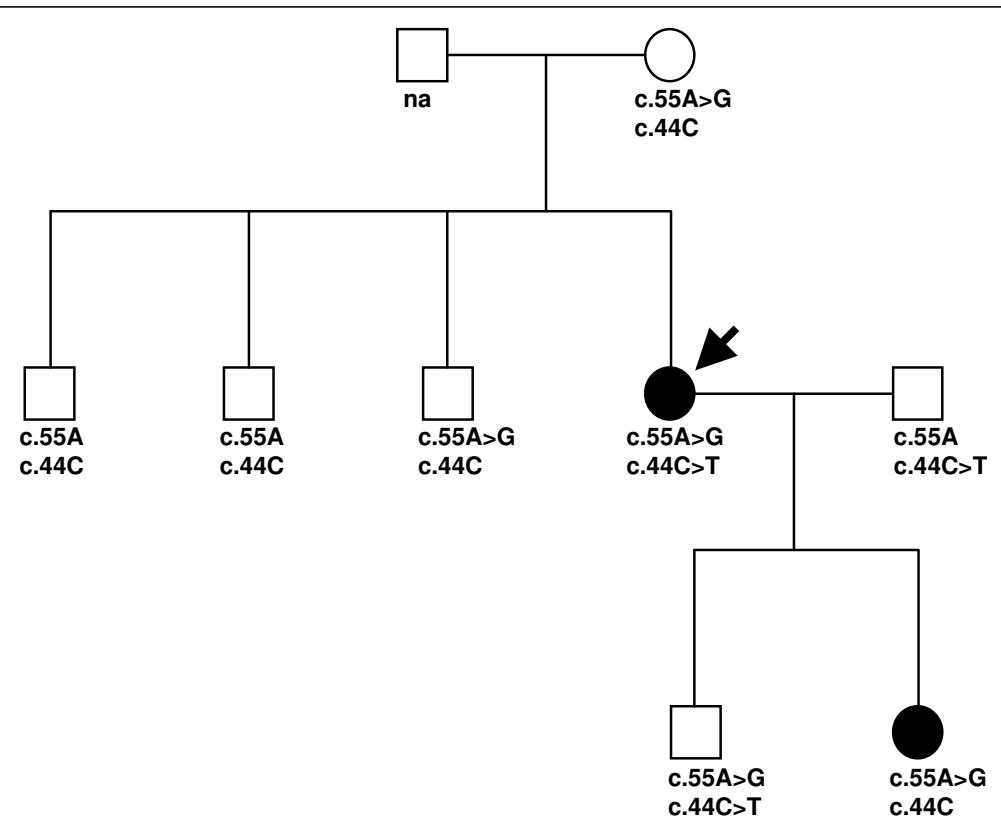

Figure 1 Pedigree of a family with Marfan syndrome associated with c.44C > T (p.S15F) and c.55A > G (p.T19A) in the TGFBR3 gene. The Index patient is indicated by arrow. na: no DNA available.

to these variants. Taken the analysis of family members into account, both variants are apparently not diseasecausing.

In our cohort, we encountered three known and one novel intronic variant in the TGFBR3 gene. Three known intronic variants c.62-51C > T (intron 2), c.247$40 \mathrm{C}>\mathrm{T}$ (intron 3) and c. $1566+55 \mathrm{C}>\mathrm{A}$ (intron 10) along with 3'UTR variant (c."19G > A) occurred in the index cases in the same allele frequency as in control cases. However, intronic variant c.886-10A > G (intron 7) is novel and was identified in a MFS case, who was later confirmed to carry a FBN1 mutation. Bioinformatic analyses using the online-programs Mutation Taster,

Table 2 Amino acid sequence comparison (44C > T; S15F and 55A > G; T19A) of the highly conserved TGFBR3 signal domain from Homo sapiens (accession no. NP_003234.2), Pan troglodytes (accession no. XP_513555.2), Sus scrofa (accession no. NP_999437.1), Mus musculus (accession no. NP_035708) and Rattus norvegicus (accession no. NP_058952.1)

\begin{tabular}{ll}
\hline Species & Amino acid sequence \\
\hline H. sapiens & SCLATAGPEP \\
P. troglodytes & $\underline{\mathbf{S}}$ CLATAGPEP \\
M. mulatta & $\underline{\mathbf{S}}$ CLATAGPEP \\
S. scrofa & $\underline{\mathbf{s}}$ CLATAGPEP \\
M. musculus & ACLATAGPEP \\
R. norvegicus & ACLA $\underline{\mathbf{T}}$ AGPEP \\
\hline
\end{tabular}

Amino acid residues found to show variation as identified in this study are highlighted in bold and respective conserved amino acids are shown in bold and underlined.
Fruitfly and NetGene2 Server did not assign any disease-causing effect to these variants.

The only novel exonic variant c.55A > G (p.T19A; exon 2) was identified in two index cases with positive family history. The first index case was a 34-year-old male with marfanoid habitus and aortic aneurysm. The affected maternal uncle of this index case who also had Marfanoid habitus and aortic aneurysm, was wild type for c.55A > G but carried another variant c. $44 \mathrm{C}>\mathrm{T}$ (p. S15F; exon 2). The mother of the index patient had a marfanoid habitus as the only symptom of MFS and did not carry c.55A > G. A healthy sister of the index case carried c.55A $>\mathrm{G}$ and the son of the deceased daughter of the maternal uncle did not carry c.55A $>$ G.

Another index patient with c.55A > G was a 40-yearold female with a mild dilatation of the aortic root (3.5 $\mathrm{cm})$, when she was a young adult. As the dilatation was not progressive, the diameter of the aortic root was in the normal range when she got older. She had skeletal involvement (arm-span to height ratio $>1.05$, positive thumb and wrist signs, flat feet, highly arched palate) and had a history of two spontaneous pneumothoraxes. She had frequent nasal bleeding and easy bruising without trauma or varicosis. The affected daughter, who carried variant c.55A > G was 8 years of age when examined and had a dilatation of the aortic root with a diameter of $2.5 \mathrm{~cm}$. Skeletal system was involved (positive thumb and wrist signs, flat feet and joint hypermobility). Additionally she had muscular hypotonia. A healthy son, brother and the mother of the index patient 
also carried c.55A $>$ G. The healthy son of the index patient carried another variant c.44C $>\mathrm{T}$ as well. Two other healthy brothers and the husband did not carry the c. $55 \mathrm{~A}>\mathrm{G}$ variant, but the husband carried the c. $44 \mathrm{C}>\mathrm{T}$ variant (figure 1). Both of these exonic variants occurred in a highly conserved TGFBR3 signal domain (table 2). A possible interpretation of c.55A > G; T19A is that it may be a predisposing factor to the aortic dilatation, as it affects a highly conserved signal domain (http://www.uniprot.org/uniprot/Q03167) and plausibly could affect the function of TGFBR3. c.55A > G; T19A may thus act as a mutation with reduced penetrance or perhaps as a variant that in combination with variation in other genes could lead to aortic dilatation. The bioinformatic prediction tool (http://www.cbs.dtu. $\mathrm{dk} /$ services/SignalP/) showed, however, that both c.55A $>\mathrm{G}$ sequences were predicted to be a valid signal sequences.

\section{Conclusions}

Taken together our data demonstrate that at least in our cohort, variations in TGFBR3 gene do not appear to play a role in the aetiology of MFS or related phenotypes, although the role of TGFBR3 variants as a genetic modifier can not be ruled out. Identification of known and novel variants in the current study could be useful in the studies of the other related disease aetiopathogeneses.

\section{Materials and Methods Probands}

49 unrelated individuals used in this study had been referred between 1997 and 2005 to our clinic or genetic testing service with suspected Marfan syndrome or fulfilling Ghent diagnostic criteria of Marfan syndrome. These patients, had already been screened for 65 along with additionally three 5 ' alternatively spliced exons of FBN1 gene, 8 exons of TGFBR2 gene, and all 9 exons of TGFBR1 gene as described before [11-14] and were found not to carry a disease-causing mutation. Blood samples were taken and genomic DNA was extracted using standard protocols. Primers were designed based on the human sequence (accession number AY796304.1) for all 17 exons of TGFBR3 gene (table 3). To analyse the exonic variants we used the bioinformatic prediction programs Mutation Taster (http:// www.mutationtaster.org/), PMut (http://mmb2.pcb.ub. es:8080/PMut/) and PolyPhen2 (http://genetics.bwh.harvard.edu/pph2/). All intronic variants and the variant in the 3'UTR were analysed with Mutation Taster (http:// www.mutationtaster.org/), Berkeley Drosophila Genome Project "Splice Site Prediction" (http://www.fruitfly.org/ seq_tools/splice.html) and NetGene2 Server (http:// www.cbs.dtu.dk/services/NetGene2/). Patients carrying
Table 3 Sequences of primer pairs used for amplification of all 17 exons and the 3'UTR of TGFBR3 gene

\begin{tabular}{|c|c|}
\hline Name & Primer sequences \\
\hline Exon $1 \mathrm{~F}$ & 5'- AGG-GAG-GGC-GAG-TGC-GCC-GGG-T-3' \\
\hline Exon $1 \mathrm{R}$ & 5'- GGA-GGT-CCT-GGC-GGC-TGG-AGC-G-3' \\
\hline CDS $1 \mathrm{~F}$ & 5'- GTC-TGT-GCT-CTG-AGC-AGC-CTG-AAG-3' \\
\hline CDS $1 \mathrm{R}$ & 5'- TCA-TCT-CAA-CTA-AAG-AGA-CTG-GGA-3' \\
\hline CDS $2 \mathrm{~F}$ & 5'- GGC-ATC-TCT-GGT-GGG-TTG-GCA-GTG-3' \\
\hline CDS $2 R$ & 5'- GCA-GAC-TCA-GTG-GCA-GTG-GGC-TGA-G-3' \\
\hline CDS 3F & 5'- GTA-TTC-CAG-AGG-CTG-CTC-TGA-G-3' \\
\hline CDS 3R & 5'- GAC-TCT-GGC-ATT-ATT-TCA-GTG-AAA-G-3' \\
\hline CDS 4F & 5'- CTT-CGA-TTT-GAG-AAG-TAC-TTT-CTC-T-3' \\
\hline CDS 4R & 5'- AAC-AAT-TGC-CTG-TCA-TAA-ATC-AGT-C-3' \\
\hline CDS 5F & 5'- GAA-TCT-GGT-TAC-CGA-GTA-CCT-CAG-3' \\
\hline CDS 5R & 5'- TCT-CCC-TGC-CTC-AAG-TCA-AGG-AAG-3' \\
\hline CDS 6F & 5'- GAC-ACT-AGA-AAC-ATG-AAG-ACT-TGG-3' \\
\hline CDS $6 \mathrm{R}$ & 5'- GAG-CTT-AGA-GAG-TCC-AAA-GAG-GCA-G-3' \\
\hline CDS 7F & 5'- CTA-AAG-TAC-TGT-TTA-ATT-TTA-GA-3' \\
\hline CDS 7R & 5'- CAT-ATA-AGC-TGA-AAT-GAC-AGT-TCC-3' \\
\hline CDS 8F & 5'- GTG-GCC-TGG-CAT-CAA-ACA-CTG-CTG-3' \\
\hline CDS $8 \mathrm{R}$ & 5'- CAG-ATG-CAG-ACT-AGG-GCC-AGA-TGG-3' \\
\hline CDS 9F & 5'- GTG-TCA-ATT-ATA-CAA-CAG-AAC-TGC-3' \\
\hline CDS $9 \mathrm{R}$ & 5'- CCC-TCT-TCA-TCT-TCA-AAG-AAA-TGT-T-3' \\
\hline CDS 10F & 5'- GAA-CCA-AAC-ACA-CAT-GGT-TTG-GTG-3' \\
\hline CDS $10 \mathrm{R}$ & 5'- GAT-AGT-CCC-TAA-CTA-AAG-CCA-ACA-A-3' \\
\hline CDS $11 \mathrm{~F}$ & 5'- ATC-CTT-CAT-ATG-ACT-GTC-ATT-AAT-C-3' \\
\hline CDS 11R & 5'- GTA-TIT-TAG-CTG-ATG-TCT-AAG-GAA-C-3' \\
\hline CDS $12 \mathrm{~F}$ & 5'- CCT-AAA-GTG-AAA-GTG-AGA-TGC-TAA-C-3' \\
\hline CDS $12 R$ & 5'- CCT-CAC-CTA-AAA-ATG-CCA-AAA-TAA-C-3' \\
\hline CDS $13 \mathrm{~F}$ & 5'- GTA-GAG-CTG-GTG-AAG-GCA-CTT-TTG-3' \\
\hline CDS 13R & 5'- GGT-CTT-CTT-AAC-AAG-CAG-AGC-TCA-G-3' \\
\hline CDS 14F & 5'- ATC-ATT-GAC-AGA-GCT-TTC-TCA-CAG-T-3' \\
\hline CDS $14 \mathrm{R}$ & 5'- GAA-TGA-GAG-CAG-AAG-TCT-CCT-TAT-C-3' \\
\hline CDS $15 \mathrm{~F}$ & 5'- TGC-AAT-GCA-TGA-TGC-AGA-CTA-ACC-A-3' \\
\hline CDS $15 R$ & 5'- ACA-AGC-TGT-TCA-CCA-ACT-CTT-ACT-C-3' \\
\hline CDS $16 \mathrm{~F}$ & 5'- GGA-ATG-CAC-ATA-CAT-AAT-ATG-CGT-C-3' \\
\hline CDS $16 \mathrm{R}$ & 5'- GAA-TAC-AAC-GGG-TGA-TCT-TTA-TAC-3' \\
\hline
\end{tabular}

TGFBR3 variants were re-contacted in order to be checked for MFS, LDS related and/or additional symptoms.

\section{PCR and DNA Sequencing}

Standard PCR conditions were initial denaturation at $95^{\circ}$ C for $10 \mathrm{~min}$ followed by 33 cycles of $96^{\circ} \mathrm{C}$ for $1 \mathrm{~min}$, $55^{\circ} \mathrm{C}$ for $1 \mathrm{~min}$ and $72^{\circ} \mathrm{C}$ for $1 \mathrm{~min}$ with final elongation for $10 \mathrm{~min}$ at $72^{\circ} \mathrm{C}$ in a $50-\mu \mathrm{l}$ reaction mixture, containing 1X buffer (Qiagen, Germany), 1X Q solution (Qiagen, Germany), $20 \mathrm{pM}$ each primer and $2.5 \mathrm{U}$ Taq Polymerase (Qiagen, Germany). The annealing temperature for exon 1 and 15 were $65^{\circ} \mathrm{C}$ and $58^{\circ} \mathrm{C}$, respectively. 
PCR products were purified with ExoSAP-IT (USB, USA), and both strands were sequenced with BigDye Terminator chemistry version 1.1 by standard protocol (ABI, USA). Sequencing reactions were carried out at $96^{\circ} \mathrm{C}$ for $10 \mathrm{~s}, 50^{\circ} \mathrm{C}$ for $5 \mathrm{~s}$, and $60^{\circ} \mathrm{C}$ for 4 mins $(25$ cycles) (Biometra, Germany). The reaction mixtures were purified using DyeEx ${ }^{\mathrm{TM}}$ 2.0 Spin Kit (Qiagen, Germany) and analyzed on the ABI Genetic Analyser 3100 according to the supplier's instructions with the sequence analysis software (ABI, USA).

\section{Controls}

All sequence alterations were checked in a sample of 55 healthy control blood donors.

\section{Author details}

${ }^{1}$ Institute of Human Genetics, Hannover Medical School, Hannover, Germany. ${ }^{2}$ Division of Cardiac Surgery, St. Michael's Hospital, Toronto, Canada.

\section{Authors' contributions}

KKS carried out the molecular genetic studies, performed the sequence alignment and drafted the manuscript. BK participated in the sequence alignment. KKS, JS and MA-K conceived of the study, and participated in its design and coordination. All authors read and approved the final manuscript.

\section{Competing interests}

The authors declare that they have no competing interests.

Received: 15 September 2011 Accepted: 2 February 2012

Published: 2 February 2012

\section{References}

1. Collod G, Babron MC, Jondeau G, Coulon M, Weissenbach J, Dubourg O, Bourdarias JP, Bonaiti-Pellie C, Junien C, Boileau C: A second locus for Marfan syndrome maps to chromosome 3p24.2-p25. Nat Genet 1994, 8(3):264-268.

2. Mizuguchi T, Collod-Beroud G, Akiyama T, Abifadel M, Harada N, Morisaki T, Allard D, Varret M, Claustres M, Morisaki $H$, et al: Heterozygous TGFBR2 mutations in Marfan syndrome. Nat Genet 2004, 36(8):855-860.

3. Singh KK, Rommel K, Mishra A, Karck M, Haverich A, Schmidtke J, ArslanKirchner M: TGFBR1 and TGFBR2 mutations in patients with features of Marfan syndrome and Loeys-Dietz syndrome. Hum Mutat 2006, 27(8):770-777.

4. Loeys BL, Chen J, Neptune ER, Judge DP, Podowski M, Holm T, Meyers J, Leitch CC, Katsanis N, Sharifi N, et al: A syndrome of altered cardiovascular, craniofacial, neurocognitive and skeletal development caused by mutations in TGFBR1 or TGFBR2. Nat Genet 2005, 37(3):275-281.

5. Loeys BL, Schwarze U, Holm T, Callewaert BL, Thomas GH, Pannu H, De Backer JF, Oswald GL, Symoens S, Manouvrier S, et al: Aneurysm syndromes caused by mutations in the TGF-beta receptor. N Engl J Med 2006, 355(8):788-798

6. Neptune ER, Frischmeyer PA, Arking DE, Myers L, Bunton TE, Gayraud B, Ramirez F, Sakai LY, Dietz HC: Dysregulation of TGF-beta activation contributes to pathogenesis in Marfan syndrome. Nat Genet 2003 33(3):407-411.

7. Byers PH: Determination of the molecular basis of Marfan syndrome: a growth industry. J Clin Invest 2004, 114(2):161-163.

8. Pepin MC, Beauchemin M, Collins C, Plamondon J, O'Connor-McCourt MD Mutagenesis analysis of the membrane-proximal ligand binding site of the TGF-beta receptor type III extracellular domain. FEBS Lett 1995, 377(3):368-372.

9. Cheifetz S, Bassols A, Stanley K, Ohta M, Greenberger J, Massague J: Heterodimeric transforming growth factor beta. Biological properties and interaction with three types of cell surface receptors. $J$ Biol Chem 1988, 263(22):10783-10789.

10. Lopez-Casillas F, Wrana JL, Massague J: Betaglycan presents ligand to the TGF beta signaling receptor. Cell 1993, 73(7):1435-1444.

11. De Paepe A, Devereux RB, Dietz HC, Hennekam RC, Pyeritz RE: Revised diagnostic criteria for the Marfan syndrome. Am J Med Genet 1996, 62(4):417-426.

12. Rommel K, Karck M, Haverich A, von Kodolitsch Y, Rybczynski M, Muller G, Singh KK, Schmidtke J, Arslan-Kirchner M: Identification of 29 novel and nine recurrent fibrillin-1 (FBN1) mutations and genotype-phenotype correlations in 76 patients with Marfan syndrome. Hum Mutat 2005, 26(6):529-539.

13. Rommel K, Karck M, Haverich A, Schmidtke J, Arslan-Kirchner M: Mutation screening of the fibrillin-1 (FBN1) gene in 76 unrelated patients with Marfan syndrome or Marfanoid features leads to the identification of 11 novel and three previously reported mutations. Hum Mutat 2002, 20(5):406-407.

14. Singh KK, Shukla PC, Rommel K, Schmidtke J, Arslan-Kirchner M: Sequence variations in the $5^{\prime}$ upstream regions of the FBN1 gene associated with Marfan syndrome. Eur J Hum Genet 2006, 14(7):876-879.

doi:10.1186/1477-5751-11-9

Cite this article as: Singh et al:: TGFBR3 variation is not a common cause of Marfan-like syndrome and Loeys-Dietz-like syndrome. Journal of Negative Results in BioMedicine 2012 11:9.

\section{Submit your next manuscript to BioMed Central and take full advantage of:}

- Convenient online submission

- Thorough peer review

- No space constraints or color figure charges

- Immediate publication on acceptance

- Inclusion in PubMed, CAS, Scopus and Google Scholar

- Research which is freely available for redistribution 